\title{
Transistor effects and in situ STM of redox molecules at room temperature
}

\author{
Albrecht, Tim; Guckian, A; Vos, JG; Ulstrup, Jens
}

Published in:

I E E E Transactions on Nanotechnology

Link to article, DOI:

10.1109/TNANO.2005.851280

Publication date:

2005

Document Version

Publisher's PDF, also known as Version of record

Link back to DTU Orbit

\section{Citation (APA):}

Albrecht, T., Guckian, A., Vos, JG., \& Ulstrup, J. (2005). Transistor effects and in situ STM of redox molecules at room temperature. I E EE Transactions on Nanotechnology, 4(4), 430-434.

https://doi.org/10.1109/TNANO.2005.851280

\section{General rights}

Copyright and moral rights for the publications made accessible in the public portal are retained by the authors and/or other copyright owners and it is a condition of accessing publications that users recognise and abide by the legal requirements associated with these rights.

- Users may download and print one copy of any publication from the public portal for the purpose of private study or research.

- You may not further distribute the material or use it for any profit-making activity or commercial gain

- You may freely distribute the URL identifying the publication in the public portal 


\title{
Transistor Effects and In Situ STM of Redox Molecules at Room Temperature
}

\author{
Tim Albrecht, Adrian Guckian, Jens Ulstrup, and Johannes G. Vos
}

\begin{abstract}
Inorganic transition metal complexes were identified as potential candidates for transistor-like behavior in an electrochemical scanning tunnelling microscope (STM) configuration at room temperature. The theoretical background has been established based on condensed matter charge transfer theory. It predicts a distinct increase of the tunnelling current close to the equilibrium potential, i.e., if molecular bridge states are tuned into resonance with the Fermi levels of the enclosing electrodes. The complexes display robust electrochemistry on $\mathrm{Au}(111)$ electrode surfaces. STM images at molecular resolution reveal detailed information on their surface structure and scanning tunnelling spectroscopy experiments have shown clear evidence of transistor-like behavior.
\end{abstract}

Index Terms-Charge transfer, conductivity, molecular electronics, resonant tunneling transistors, transition metal compounds.

\section{INTRODUCTION}

$\mathbf{O}$ BJECTIVES of emerging nanoscale chemistry at semiconductor and metal surfaces are to map and control the twoor even three-dimensional structure of molecular entities ranging from small organic molecules to transition metal complexes and larger architectures such as proteins, DNA, and dendrimers.

With a view on molecular electronics such as molecular rectification and transistor effects, there is particular interest in understanding and controlling the electronic coupling between the molecular bridge states and electronic levels around the Fermi levels of the enclosing electrodes. This coupling is essentially what induces the desirable pronounced switching effects between "on" and "off" states.

Within the last few years, these concepts have been described both theoretically [1, refs. [7], [8], [10], and [19]-[23] therein] and experimentally [2]-[4] in increasing detail. As a result, functional devices such as switches, rectifiers, diodes, and transistors seem to be within reach. Most studies thus far have, however, concentrated on ultrahigh vacuum, air, or nonconducting low dielectric-constant solvent environments where the target molecules are enclosed in a two-electrode configuration. This leaves the bias voltage as the sole external controlling factor. Molecules that have shown molecular switching effects in the form of resonance tunnelling or negative differential

Manuscript received July 15, 2004; revised December 1, 2004. This work was supported by the European Union Network SUSANA under Contract HPRN-CT-2002-00185, by the NanoScience Center, University of Copenhagen, and by the Danish Technical Science Research Council.

T. Albrecht and J. Ulstrup are with the Department of Chemistry, Technical University of Denmark, Dk-2800 Lyngby, Denmark.

A. Guckian and J. G. Vos are with the School of Chemical Sciences, Dublin City University, Dublin 9, Ireland.

Digital Object Identifier 10.1109/TNANO.2005.851280 resistance features include organic aromatic thiols [5], aminoand nitro-substituted ohenylene ethylenes [6], ferrocene-based molecular entities [7], [8], and phthalocyanines [9], all at $\mathrm{Au}$ surfaces in scanning tunnelling microscope (STM) configurations. Other molecular resonance tunnelling systems are organic molecules on $\mathrm{Si}$ surfaces [10], [11] and heteropolytungstate anions on highly oriented pyrolytic graphite [12]. Recent cases of single-moolecule transistor or amplifier effects of Coulomb staircases, tuned by the gate electrode in three-electrode configurations, have also been reported [13], [14]. In addition to ultrahigh vacuum conditions, these systems, however, also require cryogenic temperatures to operate. On the other hand, operation at room temperature is clearly needed in the approach of new monolayer and single-molecule technologies.

We propose a different approach to investigate switching effects in small molecular entities. It relies on similarities between a three-electrode transistor and a three-electrode electrochemical in situ STM configuration, i.e., in an electrochemical environment at room temperature [15]. In such a setup, the analog of source, drain, and gate contacts are the working electrode, STM tip, and reference electrode, respectively. Atconstant bias, molecular bridge states can be tuned intoresonance with the Fermi levels of the tip and working electrode by changing the working electrode potential relative to the reference potential, while the tunnelling current is monitored at a constant bias voltage [scanning tunnelling spectroscopy (STS)] [16], [17]. According to charge transfer theory adapted to such a configuration [18]-[20], the tunnelling current will show a peak feature once resonance is reached, resulting in the desired transistor effect. This resonance is, moreover, expected to be close to the equilibrium redox potential of a particular state if the bias voltage is small.

We have identified inorganic transition metal complexes with $\mathrm{Os}, \mathrm{Co}$, and $\mathrm{Ru}$ as central ions to be promising candidates for testing these theoretical expectations. These complexes are stable in at least two redox states with low-lying electronic states, their ligand chemistry is robust and well understood, and they can be modified relatively easily, e.g., with respect to the linker group at the metal/molecule interface. With the theoretical frame set and detailed electronic structure calculations on the way, we have obtained experimental evidence of this effect by in situ STM and STS combined with a variety of electrochemical techniques on atomically planar single-crystal gold and platinum surfaces.

\section{EXPERIMENTAL}

All chemicals were of highest available grade, $\mathrm{H}_{2} \mathrm{O}$ was supplied by a Millipore system (Milli-Q Housing). The complexes $\left[\mathrm{Os}(\mathrm{bpy})_{2}(\mathrm{p} 0 \mathrm{p})_{2}\right]\left(\mathrm{PF}_{6}\right)_{2}$ (1) and $\left[\mathrm{Os}(\mathrm{pby})_{2}(\mathrm{p} 2 \mathrm{p})_{2}\right]\left(\mathrm{PF}_{6}\right)_{2}$ (2) 
were prepared by refluxing $\left[\mathrm{Os}(\mathrm{bpy})_{2} \mathrm{Cl}_{2}\right] \cdot 2 \mathrm{H}_{2} \mathrm{O}$ and $\mathrm{AgNO}_{3}$ in a 1:1 ethylene glycol/ $\mathrm{H}_{2} \mathrm{O}$ solution until a grey precipitate was formed. After removing the precipitate, either $\mathrm{p} 0 \mathrm{p}$ or $\mathrm{p} 2 \mathrm{p}$ were added and refluxing continued for $96 \mathrm{~h}$. After removing unreacted ligand and addition of a concentrated aqueous solution of ammonium hexaphosphate, the product was washed with $\mathrm{H}_{2} \mathrm{O}$, dried with diethylether, and purified by column chromatography. The identity of the compounds was checked by NMR spectroscopy on a Bruker Avance $400(400 \mathrm{MHz})$ spectrometer (bpy $=2,2^{\prime}$-bipyridyl; p0p $=4,4^{\prime}$-bipyridyl; p2p $=1,2$-bis(-4-pyridyl)ethane).

$\mathrm{Au}(111)$ electrodes were prepared as published before [21]. For STM, an $\mathrm{Au}(111)$ disc electrode $(d=10 \mathrm{~mm})$ was purchased from Surface Preparation Laboratory, Zaandam, The Netherlands. Electrodes were annealed in an $\mathrm{H}_{2}$-flame, cooled in $\mathrm{H}_{2} \mathrm{O}$-saturated air, and transferred to the stock solution of a particular complex immediately.

The stock solutions were prepared in a concentration range of $10-100 \mu \mathrm{M}$ in a mixture of $\mathrm{H}_{2} \mathrm{O} / \mathrm{DMF} 2: 1(\mathrm{~m} / \mathrm{m})$. Electrodes were soaked overnight, then rinsed with Millipore $\mathrm{H}_{2} \mathrm{O}$ and electrolyte $\left(0.1 \mathrm{M} \mathrm{HClO}_{4}\right)$.

An electrochemical Autolab system controlled by a GPES software package (Ecochemie, Utrecht, The Netherlands) was used including custom-made electrochemical (glass) cells. A freshly prepared reversible hydrogen electrode (RHE) and a Pt wire served as the reference and counter electrode, respectively. The reference potential was checked with a saturated calomel electrode (SCE) after each experiment. The $\mathrm{Au}(111)$ electrodes were employed in a hanging-meniscus configuration. Prior to each experiment, the electochemical cell was deoxygenated by purified $\mathrm{Ar}$ (Chrompack $\mathrm{O}_{2}$ filter) and a steady flow of $\mathrm{Ar}$ was maintained at all times during operation.

A PicoSPM instrument (Molecular Imaging, Tempe, AZ) with a bipotentiostat for independent control of substrate and tip potential was used in a constant-current mode. The custom-made three-electrode Teflon cell was equipped with two Pt wires serving as the reference and counter electrodes. The reference potential was checked with an SCE reference electrode after each experiment. The concentration of either (1) or (2) in the electrolyte was in the range of $1-10 \mu \mathrm{M}$.

\section{THEORY}

The theoretical background of room-temperature molecular transistor functionality is described in the literature [1, refs. [7], [8], [10], and [19]-[23] therein], [18]-[20]. In the fully adiabatic case where both electron transfer steps, working electrode/bridge, and bridge/tip accord with the adiabatic limit of strong coupling between the molecule and the enclosing electrodes, the tunnelling current can be described by (1) assuming two symmetric metal contacts

$$
\begin{array}{r}
i_{\text {tunn }}^{\mathrm{ad}}=\frac{1}{2} \cdot e \kappa \rho\left(e V_{\text {bias }}\right) \cdot \frac{\omega_{\text {eff }}}{2 \pi} \cdot \exp \left(-\frac{E_{R}+e V_{\text {bias }}}{4 k_{B} T}\right) \\
\times \cosh ^{-1}\left[\frac{\left(\frac{1}{2}-\gamma\right) e V_{\text {bias }}-e \xi \eta}{2 k_{B} T}\right]
\end{array}
$$

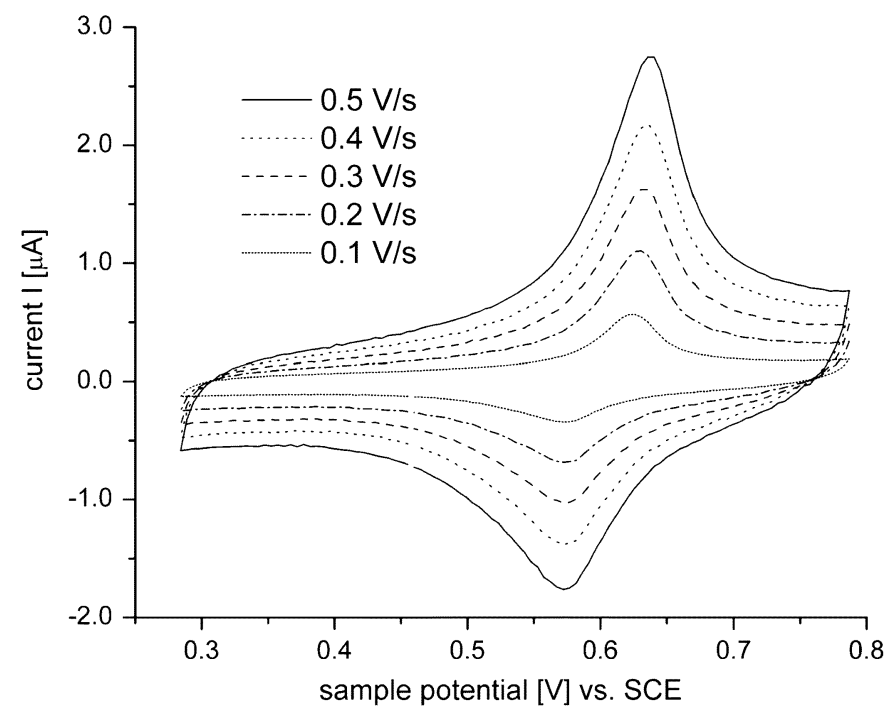

Fig. 1. CVs of compound (1) at different scan rates $\nu$ from 0.1 to $0.5 \mathrm{~V} / \mathrm{s}$, electrolyte: $0.1 \mathrm{M} \mathrm{HClO}_{4}$.

where $\kappa$ is the transmission coefficient, $\rho$ is the level density in the two metals, $\omega_{\text {eff }}$ is a characteristic (nuclear) frequency, $E_{R}$ is the molecular and environmental reorganization free energy, $\gamma$ and $\xi$ are the fractional potential drops of bias and sample potential at the redox center, and $\eta$ is the overpotential, i.e., the difference between the equilibrium and sample potentials. All other parameters have their usual meaning.

If the bias potential drop $\gamma$ at the redox center equals one-half, the maximum of the tunnelling current is found at $\eta=0$, i.e., at the equilibrium potential. By modulating the working electrode potential, molecular redox levels can thus be tuned in and out of resonance, resulting in an "on" and "off" state analogous to a transistor-like device. For a detailed description of the electronic properties of such a device, theoretical frames need to be substantiated by experimental data, specifically regarding the metal/bridge interface and the central metal ion.

\section{EXPERIMENTAL RESULTS}

Prior to any in situ STM experiment, the stability of the electrochemical response from complexes (1) and (2) and a corresponding potential window was characterized by cyclovoltammetry on single-crystal $\mathrm{Au}(111)$ electrodes, as shown in Fig. 1.

A plot of the peak current $I_{p}$ versus the scan rate $\nu$ yielded a linear correlation, indicating that the observed electron transfer process indeed originates from a surface-confined species (not shown). The electrochemical response was stable for at least several hundred potential scans.

In situ STM investigations under the same conditions have revealed that the monolayers of both compounds (1) and (2) consist of two different domain types.

The major part of the electrode surface seems to be disordered, but some areas display highly ordered structures where images of molecular resolution were obtained. Fig. 2 shows a high-resolution in situ STM image of compound (2).

A double-striped feature is clearly visible, which is composed of two individual lines each displaying molecular substructure. The length of such an individual line is approximately $19 \AA$. 


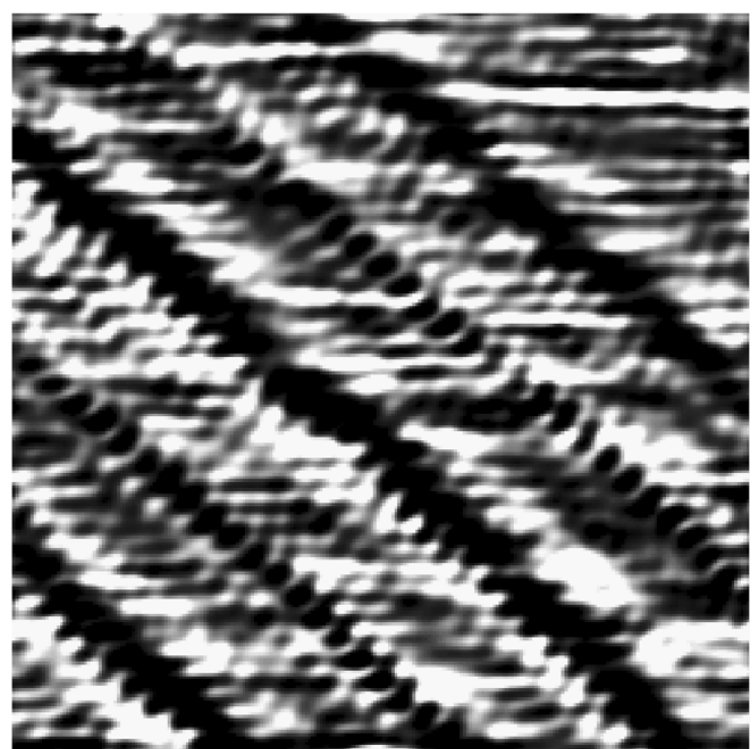

Fig. 2. In situ STM image of compound (2) in $0.1 \mathrm{M} \mathrm{HClO}_{4}$. Area: $(12.5 \mathrm{~nm})^{2}$, $E_{s}=+0.25 \mathrm{~V}, V_{\text {bias }}=-0.2 \mathrm{~V}, I_{\text {set }}=50 \mathrm{pA}$ (all potentials versus SCE). Tip material: W.

This is very similar to the length of (2) along one of the linker ligands p2p (ca. $17 \AA$ ). We, therefore, conclude that, at least in the highly ordered domains, only one ligand is bound to the surface, while the other one points into the solution, presumably being protonated. This finding is in line with previous work by Forster et al. who came to the same conclusion for a comparable complex $\left[\mathrm{Os}(\mathrm{bpy})_{2}(\mathrm{p} 3 \mathrm{p})_{2}\right]\left(\mathrm{PF}_{6}\right)_{2}$, based on electrochemical data [22].

After repetitive cycling of the sample potential in the range shown in Fig. 1, the ordered domains disappear and only the disordered phase is observed.

In order to probe the dependence of the tunnelling current $I_{t}$ on the sample potential $E_{s}$ for compound (2), the feedback loop was switched off. With the tip still in the tunnelling range, $E_{s}$ was cycled from -0.05 to $+0.8 \mathrm{~V}$ at a constant bias voltage and $I_{t}$ was monitored. A typical sweep lasted $10 \mathrm{~s}$ and only those scans were included in the data evaluation where the tunnelling current only changed insignificantly before and after a potential sweep (minimal vertical drift). From in situ STM imaging, the lateral drift was estimated to be around $10 \AA$ during a potential sweep.

The result of averaging 20 subsequent in situ STS scans is shown in Fig. 3, together with a cyclovoltammogram (CV) of (2).

The in situ STS curve shows a clear peak-like feature around the equilibrium potential of the complex.

Upon retracting the tip from the surface, i.e., out of the tunnelling range, the peak vanishes completely, showing that the observed feature indeed refers to a resonant tunnelling effect. Additional experiments on bare $\mathrm{Au}(111)$, with a different tip material $(\mathrm{W})$ and electrolyte $\left(\mathrm{HNO}_{3}\right)$ have supported this interpretation. It could be shown that purely Faradaic effects, such as the oxidation/reduction of a bare gold surface, do not cause any change in the tunnelling current (not shown).

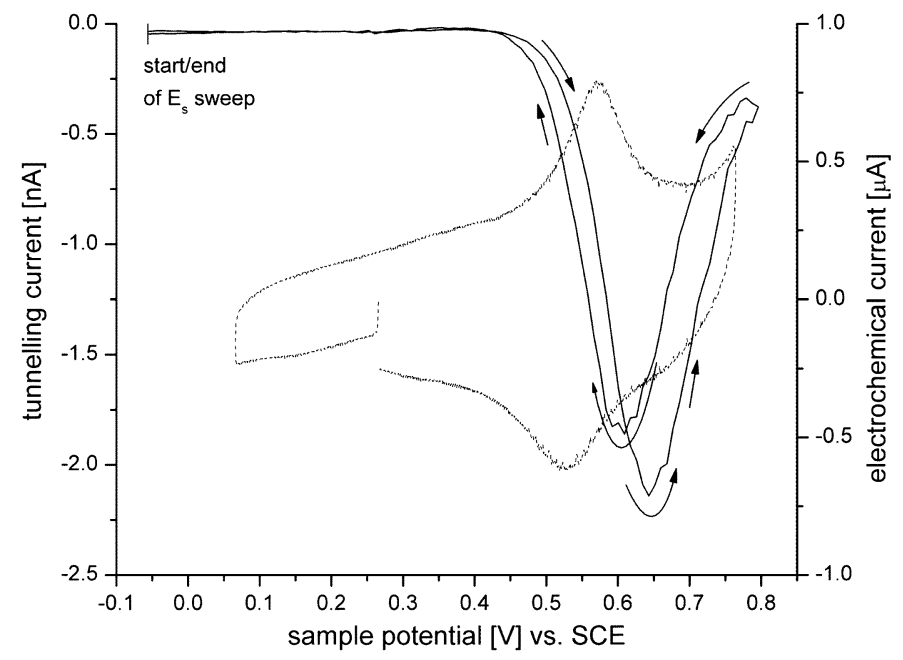

Fig. 3. In situ STS curve (solid line, left-hand-side ordinate) and CV (dashed line, right-hand-side ordinate) of compound (2) in 0.1-M HClO ${ }_{4}$. STS: $E_{s}$ scan range from -0.05 to $+0.8 \mathrm{~V}, V_{\text {bias }}=-0.1 \mathrm{~V}$. Sweep duration: $10 \mathrm{~s}$. Tip material: Pt/Ir (80/20). CV: $\nu=0.1 \mathrm{~V} / \mathrm{s}$ (all potentials versus SCE). Note the different ordinate scales.

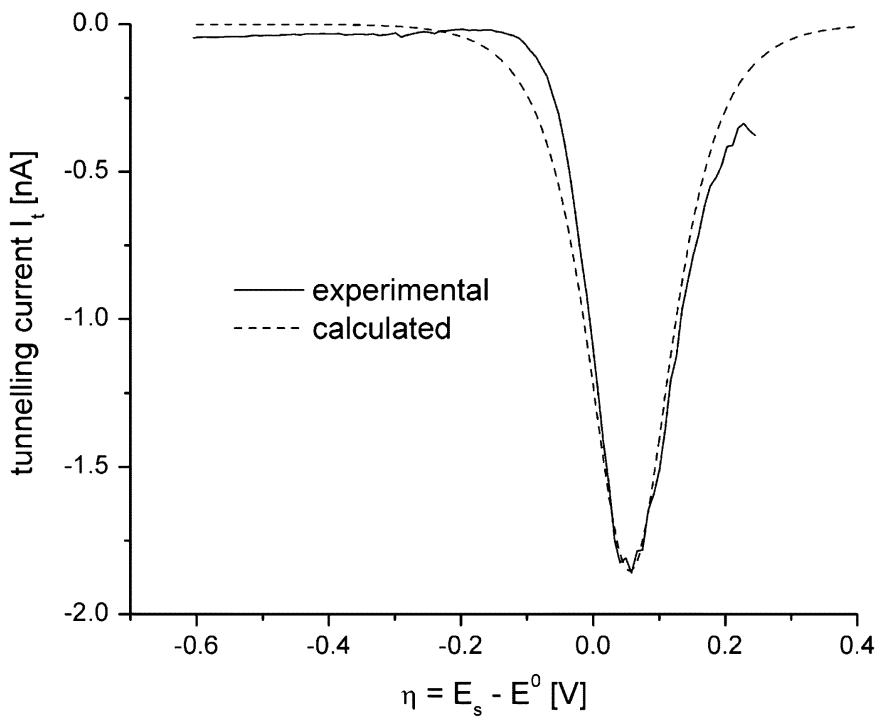

Fig. 4. Experimental and simulated in situ STS correlations according to (1) (solid and dashed lines, respectively). $E_{R}=0.5 \mathrm{eV}, V_{\text {bias }}=-0.1 \mathrm{~V}, \gamma=$ $1, \xi=0.9, \kappa \cdot \rho \cdot \omega_{\text {eff }} \cdot e^{2} /(4 \cdot \pi)=9.1 \cdot 10^{-7} \mathrm{C}^{2} /(\mathrm{eV} \cdot \mathrm{s})$. Experimental data from reverse scan (from high-to-low potentials) in Fig. 3.

If the observed peak was due to Faradaic effects, the measured current should change sign upon reversing the scan direction, as in cyclovoltammetry. This is not the case.

The peak position depends slightly on the sweep direction, as shown in Fig. 3. This effect could be related to dielectric relaxation in the tunnelling gap in response to the potential sweep since the peak separation decreases with increasing sweep duration.

Independent of sweep direction, the tunnelling current peaks at a slightly higher sample potential than the electrochemical equilibrium potential in CV. This is, however, in accordance with (1), as the immobilized redox center is located closer to the tip than to the substrate and experiences a fraction of the bias voltage $\gamma>0.5$ in addition to the sample potential. 
A simulation based on (1) shows that the adiabatic charge transfer model can indeed reproduce the peak position, shape, and, to a reasonable approximation, also its intensity (Fig. 4).

At the current stage, it is difficult to draw conclusions from the absolute intensity of the peak since the true distance between the tip, redox center, and surface is not accurately known. Both nonvanishing vertical and lateral drift in the in situ STM experiments at room temperature complicate the situation. As the observed peak is most probably due to a single or a very small number of molecules, there may also be some dispersion in their electrochemical and tunnelling properties. Its multielectronic nature will, however, lead to some temporal averaging. The actual ratio between peak and background intensity, in terms of an "on"/“off” ratio, amounts to approximately 50 (Fig. 3).

\section{CONCLUSION}

We have synthesized two compounds of a larger class of transition metal complexes identified as promising candidates for displaying transistor-like features in their molecular electronic conductivity at room temperature and in an electrochemical environment. The theoretical framework has been established. The electrochemical characterization of both compounds has shown that they form self-assembled monolayers on $\mathrm{Au}(111)$. STM images to molecular resolution have been obtained that allow for insight into details of their surface organization. Their electrochemical properties support our design strategy. Detailed STS investigations have provided evidence of resonant tunnelling effects related to the redox state of the metal complex. Further experiments are on the way to probe the electronic properties of the molecules at the single-molecule level and to elucidate the role of the central metal ion and the linker group to the electrode surface.

\section{REFERENCES}

[1] A. M. Kuznetsov and J. Ulstrup, "Single-molecule electron tunnelling through multiple redox levels with environmental relaxation," J. Electroanal. Chem., vol. 564, pp. 209-222, 2004.

[2] R. M. Metzger, "Unimolecular electrical rectifiers," Chem. Rev., vol. 103 , pp. 3803-3834, 2003.

[3] A. Aviram, M. Ratner, and V. Mujica, Eds., Molecular Electronics II. New York: New York Acad. Sci., 2002.

[4] J. G. Kushmerick et al., "Understanding charge transport in molecular electronics," in Molecular Electronics III, J. R. Reimers, C. A. Picconatto, J. C. Ellenbogen, and A. Shashidar, Eds. New York: New York Acad. Sci., 2003, vol. 1006, pp. 277-290.

[5] Y. Xue, S. Datta, S. Hong, R. Reifenberger, J. I. Henderson, and C. P. Kubiak, "Negative differential resistance in the scanning-tunneling spectroscopy of organic molecules," Phys. Rev. B, Condens. Matter, vol. 59, pp. R7852-R7855, 1999.

[6] F. F. Fan, J. Yang, L. Cai, D. W. Price, Jr., S. M. Dirk, D. V. Kosynkin, Y. Yao, A. M. Rawlett, J. M. Tour, and A. J. Bard, "Charge transport through self-assembled monolayers of compounds of interest in molecular electronics," J. Amer. Chem. Soc., vol. 124, pp. 5550-5560, 2002.

[7] C. B. Gorman, R. L. Carroll, and R. Fuierer, "Negative differential resistance in patterned electroactive self-assembled monolayers," Langmuir, vol. 17, pp. 6923-6930, 2001.

[8] R. A. Wassel, G. M. Credo, R. R. Fuierer, D. L. Feldheim, and C. B. Gorman, "Attenuating negative differential resistance in an electroactive self-assembled monolayer-based junction," J. Amer. Chem. Soc., vol. 126, pp. 295-300, 2004.

[9] L. Scudiero, D. E. Barlow, and K. W. Hipps, "Physical properties and metal ion specific scanning tunneling microscopy images of metal(II) tetraphenylporphyrins deposited from vapor onto gold(111)," J. Phys. Chem. B, vol. 104, pp. 11899-11 905, 2000.
[10] N. P. Guisinger, M. E. Greene, R. Basu, A. S. Baluch, and M. C Hersam, "Room temperature negative resistance through individual organic molecules on silicon surfaces," Nano Lett., vol. 4, pp. 55-59, 2004.

[11] — "Observed suppression of room temperature negative differential resistance in organic monolayers on $\mathrm{Si}(100)$," Nanotechnology, vol. 15, pp. S452-S458, 2004.

[12] M. S. Kaba, I. K. Song, and M. A. Barteau, "Ordered array formation and negative differential resistance behavior of cation-exchanged heteropoly acids probed by scanning tunneling microscopy," J. Phys. Chem., vol. 100, pp. 19577-19581, 1996.

[13] S. Kubatkin et al., "Single-electron transistor of a single organic molecule with access to several redox states," Nature, vol. 425, pp. 698-701, 2003.

[14] J. Park et al., "Coulomb blockade and the Kondo effect in single-atom transistors," Nature, vol. 417, pp. 722-725, 2002.

[15] D. M. Kolb, "Electrochemical surface science," Angew. Chem. Int. Ed. vol. 40, pp. 1162-1181, 2001

[16] N. J. Tao, "Probing potential-tuned resonant tunneling through redox molecules with scanning tunneling microscopy," Phys. Rev. Lett., vol. 76, pp. 4066-4069, 1996.

[17] W. Haiss et al., "Redox state dependence of single molecule conductivity," J. Amer. Chem. Soc., vol. 125, pp. 15 294-15 295, 2003.

[18] A. M. Kuznetsov and J. Ulstrup, "Mechanisms of in situ scanning tunnelling microscopy of organized redox molecular assemblies," J. Phys. Chem. A, vol. 104, pp. 11531-11 540, 2000.

[19] _ "Errata of 'Mechanisms of in situ scanning tunnelling microscopy of organized redox molecular assemblies'," J. Phys. Chem. A, vol. 105 , p. 7494, 2001.

[20] J. Zhang et al., "Electronic properties of functional biomolecules at metal/aqueous solution interfaces," J. Phys. Chem. B, vol. 106, pp. 1131-1152, 2002.

[21] J. Clavilier et al., "The role of anion on the electrochemical behaviour of a 111 platinum surface: An unusual splitting of the voltammogram in the hydrogen region," J. Electroanal. Chem., vol. 107, pp. 205-209, 1980.

[22] R. J. Forster and J. P. O'Kelly, "pH modulated heterogeneous electron transfer across metal/monolayer interfaces," J. Phys. Chem., vol. 100, pp. 3695-3704, 1996.

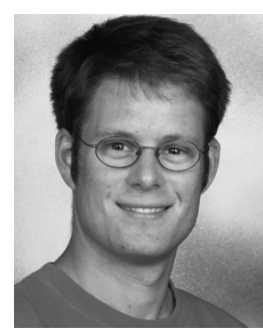

Tim Albrecht received the Diploma degree in chemistry from the University of Essen, Essen, Germany in 2000, and the Ph.D. degree from the Technical University of Berlin, Berlin, Germany, in 2003.

In 2000, he was with the University of California at Berkeley. During his doctoral studies, he was with the Max-Planck-Institute of Radiation Chemistry, Muelheim, Germany, where he focused on interfacial electron transfer of natural and artificial (de novo) heme proteins by single-crystal electrochemistry, surface-enhanced resonance Raman (SERR) spectroscopy, and in situ scanning tunneling microscopy. He also visited the New University of Lisbon, Oeiras, Portugal, and the Technical University of Denmark, Lyngby, Denmark. In 2003, he rejoined the Technical University of Denmark, as a Post-Doctoral Researcher, with a special focus on single-crystal electrochemistry and in situ scanning tunneling microscopy/spectroscopy of transition metal complexes and other nanoscopic architectures.

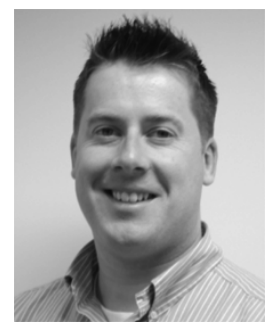

Adrian Guckian received the B.Sc. (Hons.) degree in pure and applied chemistry from Dublin City University, Dublin, Ireland, in 1998, and the Ph.D. degree from Dublin City University, Dublin, Ireland, in 2002. His dissertation was entitled "Synthesis and Characterization of a Series of Mononuclear Complexes and Surface Active Dinuclear Complexes."

In 1998, he began his research career with Dublin City University, and spent the first year of his postgraduate studies with the University of Bologna. His research has involved the synthesis of ruthenium and osmium complexes and the examination of the photophysical and photochemical properties of these complexes. Much of his research also concerned the evaluation of the electrochemical and spectroelectrochemical properties of metal complexes both in solution and while attached to surfaces. In 2002, he began a Post-Doctoral Fellowship with the National Centre for Sensor Research, Dublin City University (DCU), Dublin, Ireland. His most recent research has focused on the development of novel luminescent complexes for use as sensors for $\mathrm{O}_{2}$, $\mathrm{CO}_{2}, \mathrm{H}_{2} \mathrm{O}$, and $\mathrm{pH}$. 


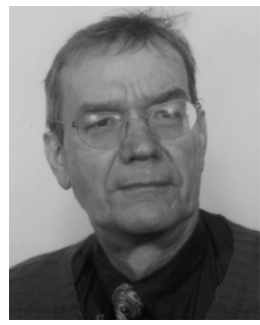

Jens Ulstrup received the degree in chemistry and D.Sc. degree from the University of Copenhagen, Copenhagen, Denmark, in 1964 and 1981, respectively.

He was an Assistant Professor and Associate Professor, and since 1984, a Full Professor of chemistry with the Technical University of Denmark, Lyngby, Denmark. He has also been a Visiting Scientist with the University of Oxford, The A. N. Frumkin Institute of Electrochemistry, the Fritz-Haber-Institut der Max-Planck-Gesellschaft, Utah State University, Forschungszentrum Jülich, and the University of Sydney. He has authored or coauthored approximately 220 research papers in major international journals, three monographs, and numerous review papers and book chapters. His research has involved chemical rate theory, charge transport in chemical, electrochemical, and biological systems, electronic processes in metalloproteins, bioinorganic chemistry, bioelectrochemistry, physical electrochemistry, and nanoscale and single-molecule properties.

Dr. Ulstrup is a member of the Danish Academy of Technical Sciences (since 1991) and the Royal Danish Academy of Sciences and Letters (since 1992). He is a Fellow of the Royal Society of Chemistry. He was the recipient of the 2003 Carlsberg Prize for chemistry and the 2004 First Bioelectrochemistry Prize of the International Society of Electrochemistry.

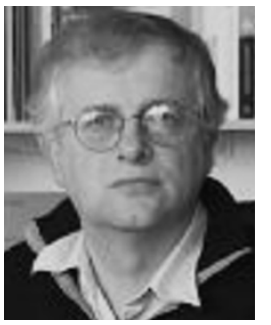

Johannes G. Vos received the Ph.D. degree in chemistry from the University of Leiden, Leiden, The Netherlands, in 1978.

He performed post-doctoral research with the University of Dublin, Trinity College (1978-1979 and 1980-1982) and the University of Fribourg, Fribourg, Switzerland (1979-1980). In 1982, he became an Assistant Lecturer with Dublin City University, Dublin, Ireland. He then became a Lecturer in 1984, a Senior Lecturer in 1987, an Associate Professor in 1994, and a Professor of inorganic chemistry in 1999, all with Dublin City University. He has authored or coauthored over 200 refereed papers, one monograph, and several book chapters. His research has involved synthesis, characterization, and properties of ruthenium and osmium containing polypyridyl compounds, energy- and electron-transfer processes in supramolecular structures, deuteriation and its effect of photophysical properties, electrochemical properties of surfaces modified with metal complexes, polymers, and carbon nanotubes.

Dr. Vos is member of the Royal Irish Academy (since 1999). 